\title{
Complete genome sequence of C130_2, a novel Myovirus infecting pathogenic Escherichia coli and
} Shigella strains

(1)

Domonkos Sváb ${ }^{1}$, Linda Falgenhauer ${ }^{2}$, Manfred Rohde $^{3}$, Trinad Chakraborty ${ }^{2}$ and

(1)

István Tóth ${ }^{1}$

(1)

${ }^{1}$ Institute for Veterinary Medical Research, Centre for Agricultural Research, Hungarian Academy of Sciences, Budapest, Hungary, ${ }^{2}$ Institute of Medical Microbiology, Justus Liebig University Giessen and German Center for Infection Research (DZIF), Partner Site Giessen-Marburg-Langen, Giessen, Germany, ${ }^{3}$ Central Facility for Microscopy, Helmholtz Centre for Infection Research, HZI, Braunschweig, Germany 1

\section{Abstract}

The genome sequence of a novel virulent bacteriophage termed C130_2 that is morphologically a member of the family Myoviridiae, is reported. The 41,775 basepair double-stranded DNA genome of C130_2 encodes for 59 ORFs but exhibits overall low sequence homology to publicly available bacteriophage genomes. Phylogenetic analyses indicates that $\mathrm{C} 130 \_2$ represents a new phage type. C130_2 propagated well on enterohemorrhagic Escherichia coli (EHEC) O157:H7 and other pathogenic E. coli strains, as well as on strains comprising various Shigella species. 
Microbial resistance against antimicrobials is an increasing problem. In recent years, researchers' attention have turned toward bacteriophages, the natural predators of bacteria, as alternative agents for use against bacteria, either post-infection as phage therapy, or preventively as bio-control agents. (reviewed in [1] and [2]).

Several members of the Enterobacteriaceae bacterial family are important enteral pathogens; many of them cause foodborne infections. In light of the serious problem of multidrug resistance, several members of the genus Enterobacteriaceae, including Escherichia coli O157:H7, Shigella species and various Salmonella serovars have been targeted in studies involving bacteriophages as biocontrol agents. Genomes of phages effective against $E$. coli O157 strains, Shigella and Salmonella representing several groups from the order Caudovirales including T4-like [3], T5-like [4], and rV5-like [5] bacteriophages have been reported. Studies testing the application of phages towards E. coli $\mathrm{O} 157: \mathrm{H} 7$ on beef [6], cabbage [7] or on tomato surfaces [8], as well as their stability under various conditions [8] were performed with promising results. Similar experiments were conducted with phages against Salmonella on duck meat [9], as well as a phage cocktail against Shigella species in various foodstuff [10].

In the current study, we present genomic characterisation of a new bacteriophage termed C130_2 isolated from cheese. This phage exhibits broad host specificity and is quite unrelated to any previously characterised bacteriophage. The phage was isolated from a cattle cheese sample from Ukraine in a project aiming to assess the risk posed by illegally imported food in the EU [11]. The phage was isolated by applying the bacterium-free supernatant of a pre-cultured food sample on layered soft agar plates containing E. coli K-12 C600 strain [4]. The host specificity of the isolated phage was investigated using a spot assay on various enterobacterial strains (Supplementary Table 1).

The efficiency of plating (EOP) was determined by applying serial dilutions of phage suspensions employing spot assays. The ratio of phage titre on the various enterobacterial strains (Supplementary Table 1) to the titre measured on E. coli K-12 MG1655 was considered as the EOP of the phage on the given strain.

The morphology of the phage examined using transmission electron microscopy (TEM). C130_2 revealed a Myoviridae morphology with an approx. $75 \times 78 \mathrm{~nm}$ icosahedral head and a $115 \mathrm{~nm}$ long contractile tail (Figure $1)$.

Phage DNA was isolated by the phenol-chloroform method [12] with the modifications outlined by Tóth et al [13]. Genomic DNA sequencing libraries were prepared using the Nextera XT kit (Illumina, Eindhoven, NL). Sequencing was performed using Nextseq Mid-output reagent kit v2 ( $2 \times 150$ bp) on an Illumina NextSeq 500 . 
Average read length was 233.39 nucleotides with an average coverage of $93.3 \%$.-Assembly was performed with Spades [14]. The genome was annotated using the RAST server [15]. A search for tRNAs was conducted with tRNAScan-SE [16]. Homology searches were performed with the tools available on the NCBI website, protein sequences of ORFs were investigated with PSI-BLAST, Prosite, and Uniprot databases. Protein masses were predicted with ExPasy using an average resolution setting.

The genome sequence of bacteriophage C130_2 was deposited in GenBank and is available under accession no. MH363708. The genome of bacteriophage C130_2 is a 41,775 bp long, linear double-stranded DNA, with a GC content of $55.4 \%$. The terminal repeats determined by a pile-up analysis of the raw reads by mapping of them to the assembled phage genome using CLC genomic workbench (v. 9.5.4, Qiagen, Venlo, Netherlands), are 284 nucleotides in length, and located distally at the $5^{`}$ and $3^{`}$ ends of the genome from nucleotides 1-284 and 41,49241,775 , respectively.

We identified a total of 59 potential protein-coding sequences (CDSs), but no tRNA genes. The list of ORFs detected is provided in Supplementary Table 2. RAST- and PSI-BLAST- based annotations enabled assignment of a function for 35 of 59 genes, with the remaining ORFs annotated as 'hypothetical', 'phage protein' or 'unknown' proteins. At the nucleotide -level, the genome does not show strong homology to any other previously sequenced bacteriophage. Whole-genome based phylogenetic relations of phage C130_2 were investigated with VICTOR [17]. This analysis placed IME_EC2 and vB_KpnS_IME279 as its closest neighbors, albeit still too far apart to be considered as close relatives. At the same time it has shown that C130_2 indeed represents a wholly new genotype within bacteriophages representing members of the order Caudovirales (Figure 2).

Prosite search detected motifs in only 8 ORFs with the inclusion of high probability occurrence motifs. Four out of these encode structural proteins, and the other four encode DNA modifying enzymes. In many cases the PSIBLAST and Uniprot searches indicated that the predicted proteins show homology to genes of Enterobacteria phage IME_EC2 (GenBank KF591601.1; [18]) and Klebsiella phage vB_KpnS_IME279 (MF614100.1). For the PSI-BLAST hits, CDSs exhibited an average coverage of $93.3 \%$ but with a low average homology of $47.4 \%$. The Uniprot hits showed $99 \%$ average coverage, and $76 \%$ average identity at the amino acid level (see Supplementary Table 2 for details). Interestingly, these two phages are members of different families, as they belong to Podoviridae and Siphoviridae, respectively. When studying the PSI-BLAST and Uniprot search results, it should be noted that except for a major tail protein (locus 130-2_0057) the majority of ORFs bearing 
similarity to corresponding ORFs in IME_EC2 or vB_KpnS_IME279 code for proteins associated with DNA modification.

A blastN-based pairwise comparison analysis was performed for the three phages C130_2, IME_EC2 and vB_KpnS_IME279 using Easyfig 2.1 [19] and visualized using Inkscape (Supplementary Figure 1). This revealed only a few regions where similarity of $\mathrm{C} 130 \_2$ to either of the other phages approaches $75 \%$, and almost never exceeds $80 \%$. The first of these regions contains two genes encoding putative proteins involved in tail assembly (ORFs 14-15). The following region spans five genes encoding DNA modification enzymes, (ORFs 28, 29, 31, 32, 33). These are followed by tail fiber, capsid, portal protein encoding genes and one that encodes the terminase large subunit (Supplementary Table 3) The relatively conserved sequence of these genes suggests their universal importance in the lifecycle of tailed bacteriophages. The order of these regions is the same in all three of the phage genomes, suggesting that their overall genome organisation is colinear (Supplementary Table 3). The rest of the C130_2 genome however, encodes for genes with as of yet unknown functions, which have a low level of similarity (below 50\%) to the other two phage genomes, indicating its novelty.

C130_2 is capable of lysing E. coli K-12, EHEC O157:H7, enteropathogenic (EPEC), enteroinvasive (EIEC) and Shigella strains with efficiency of plating (EOP) between approx.10-2 to $2 \times 10^{-8}$ (Supplementary Table 1). The fact that phage C130_2 lyses multiple Shigella strains is an important finding, as Shigellae are a leading cause of bacillary dysentery [20]. Like other significant pathogens, antibiotic resistance is a rising menace among Shigella strains [21], and promising experiments aiming the development of anti-Shigella phage cocktails have been performed [10]. For foodborne pathogens, it is desirable that bacteriophages present in the same foodstuff be considered as prime candidates in studies searching for biocontrol agents. Our study demonstrates that so far completely uncharacterised bacteriophages potentially effective against and significant foodborne pathogens are indeed present in the same foodstuff in which their hosts reside.

Whole genome sequencing of new bacteriophages may reveal hitherto unknown genes regulating host specificity, as well as those that play key roles in lysis and survival. Detailed knowledge of the host spectrum, stability and efficiency of different phages and the associated genes could help in assembling more effective phage cocktails or even the generation of specifically modified phages to be applied against different arrays of pathogenic bacteria. 


\section{Acknowledgments}

109

110

111

112

113

The study was supported by the Hungarian National Research, Development and Innovative Office (NKFIH, grant 124335), 7th EU Framework Programme PROMISE project (project n. 265877) and by grants to the German Center of Infection Research (DZIF, site Giessen-Marburg-Langen), through the German Federal Ministry of Education and Research, grant \# TI06.001 and 8032808811 to TC. We thank Christina Gerstmann for excellent technical assistance. Domonkos Sváb was supported by the János Bolyai Research Scholarship of the Hungarian Academy of Sciences.

\section{References}

2. Abedon ST (2017) Bacteriophage clinical use as antibacterial "drugs": Utility and Precedent. Microbiol Spectr 5: . doi: 10.1128/microbiolspec.BAD-0003-2016

3. Hong Y, Pan Y, Harman NJ, Ebner PD (2014) Complete genome sequences of two Escherichia coli O157:H7 phages effective in limiting contamination of food products. Genome Announc 2: . doi: 10.1128/genomeA.00519-14

4. Sváb D, Falgenhauer L, Rohde M, et al (2018) Identification and characterization of T5-like bacteriophages representing two novel subgroups from food products. Front Microbiol 9:202 . doi: 10.3389/fmicb.2018.00202

5. Kim M, Heu S, Ryu S (2014) Complete genome sequence of enterobacteria phage 4MG, a new member of the subgroup "PVP-SE1-like phage" of the "rV5-like viruses." Arch Virol 159:3137-3140 . doi: $10.1007 / \mathrm{s} 00705-014-2140-1$

6. Liu H, Niu YD, Meng R, et al (2015) Control of Escherichia coli O157 on beef at 37, 22 and $4^{\circ} \mathrm{C}$ by T5-, T1-, T4-and O1-like bacteriophages. Food Microbiol 51:69-73 . doi: 10.1016/j.fm.2015.05.001

7. Lee H, Ku H-J, Lee D-H, et al (2016) Characterization and genomic study of the novel bacteriophage HY01 infecting both Escherichia coli O157:H7 and Shigella flexneri: Potential as a biocontrol agent in food. PloS One 11:e0168985 . doi: 10.1371/journal.pone.0168985

8. Ramirez K, Cazarez-Montoya C, Lopez-Moreno HS, Castro-Del Campo N (2018) Bacteriophage cocktail for biocontrol of Escherichia coli O157:H7: Stability and potential allergenicity study. PloS One 13:e0195023 . doi: 10.1371/journal.pone.0195023

9. Wang C, Chen Q, Zhang C, et al (2017) Characterization of a broad host-spectrum virulent Salmonella bacteriophage fmb-p1 and its application on duck meat. Virus Res 236:14-23 . doi: 10.1016/j.virusres.2017.05.001

10. Soffer N, Woolston J, Li M, et al (2017) Bacteriophage preparation lytic for Shigella significantly reduces Shigella sonnei contamination in various foods. PloS One 12:e0175256 . doi: 10.1371/journal.pone.0175256

11. Nagy B, Szmolka A, Smole Možina S, et al (2015) Virulence and antimicrobial resistance determinants of verotoxigenic Escherichia coli (VTEC) and of multidrug-resistant E. coli from foods of animal origin 
illegally imported to the EU by flight passengers. Int J Food Microbiol 209:52-59 . doi: 10.1016/j.ijfoodmicro.2015.06.026

12. Sambrook J, Maniatis T, Fritsch EF, Laboratory CSH (1987) Molecular cloning : a laboratory manual, 2nd ed. Cold Spring Harbor, N.Y. : Cold Spring Harbor Laboratory Press

13. Tóth I, Sváb D, Bálint B, et al (2016) Comparative analysis of the Shiga toxin converting bacteriophage first detected in Shigella sonnei. Infect Genet Evol J Mol Epidemiol Evol Genet Infect Dis 37:150-157 . doi: 10.1016/j.meegid.2015.11.022

14. Bankevich A, Nurk S, Antipov D, et al (2012) SPAdes: A new genome assembly algorithm and its applications to single-cell sequencing. J Comput Biol 19:455-477 . doi: 10.1089/cmb.2012.0021

15. Overbeek R, Olson R, Pusch GD, et al (2014) The SEED and the rapid annotation of microbial genomes using subsystems technology (RAST). Nucleic Acids Res 42:D206-214 . doi: 10.1093/nar/gkt1226

16. Lowe TM, Chan PP (2016) tRNAscan-SE On-line: integrating search and context for analysis of transfer RNA genes. Nucleic Acids Res 44:W54-57 . doi: 10.1093/nar/gkw413

17. Meier-Kolthoff JP, Göker M (2017) VICTOR: genome-based phylogeny and classification of prokaryotic viruses. Bioinformatics 33:3396-3404 . doi: 10.1093/bioinformatics/btx440

18. Hua Y, An X, Pei G, et al (2014) Characterization of the morphology and genome of an Escherichia coli podovirus. Arch Virol 159:3249-3256 . doi: 10.1007/s00705-014-2189-x

19. Sullivan MJ, Petty NK, Beatson SA (2011) Easyfig: a genome comparison visualizer. Bioinforma Oxf Engl 27:1009-10 . doi: 10.1093/bioinformatics/btr039

20. Anderson M, Sansonetti PJ, Marteyn BS (2016) Shigella diversity and changing landscape: Insights for the twenty-first century. Front Cell Infect Microbiol 6:45 . doi: 10.3389/fcimb.2016.00045

21. Nüesch-Inderbinen M, Heini N, Zurfluh K, et al (2016) Shigella antimicrobial drug resistance mechanisms, 2004-2014. Emerg Infect Dis 22:1083-1085 . doi: 10.3201/eid2206.152088

22. Blattner FR (1997) The complete genome sequence of Escherichia coli K-12. Science 277:1453-1462 . doi: $10.1126 /$ science.277.5331.1453

23. Hayashi T (2001) Complete genome sequence of enterohemorrhagic Eschelichia coli O157:H7 and genomic comparison with a laboratory strain K-12. DNA Res 8:11-22 . doi: 10.1093/dnares/8.1.11

24. Perna NT, Plunkett G, Burland V, et al (2001) Genome sequence of enterohaemorrhagic Escherichia coli O157:H7. Nature 409:529-533 . doi: 10.1038/35054089

25. Marchès O, Ledger TN, Boury M, et al (2003) Enteropathogenic and enterohaemorrhagic Escherichia coli deliver a novel effector called Cif, which blocks cell cycle G2/M transition. Mol Microbiol 50:1553-1567

26. Sváb D, Bálint B, Maróti G, Tóth I (2016) Cytolethal distending toxin producing Escherichia coli O157:H43 strain T22 represents a novel evolutionary lineage within the O157 serogroup. Infect Genet Evol J Mol Epidemiol Evol Genet Infect Dis. doi: 10.1016/j.meegid.2016.11.003

27. Iguchi A, Thomson NR, Ogura Y, et al (2009) Complete genome sequence and comparative genome analysis of enteropathogenic Escherichia coli O127:H6 strain E2348/69. J Bacteriol 191:347-354 . doi: 10.1128/JB.01238-08

28. Hochhut B, Wilde C, Balling G, et al (2006) Role of pathogenicity island-associated integrases in the genome plasticity of uropathogenic Escherichia coli strain 536. Mol Microbiol 61:584-595 . doi: $10.1111 / \mathrm{j} .1365-2958.2006 .05255 . \mathrm{x}$ 
29. Moriel DG, Bertoldi I, Spagnuolo A, et al (2010) Identification of protective and broadly conserved vaccine antigens from the genome of extraintestinal pathogenic Escherichia coli. Proc Natl Acad Sci U S A 107:9072-9077 . doi: 10.1073/pnas.0915077107

30. Tóth I, Nougayrède J-P, Dobrindt U, et al (2009) Cytolethal distending toxin type I and type IV genes are framed with lambdoid prophage genes in extraintestinal pathogenic Escherichia coli. Infect Immun 77:492-500 . doi: 10.1128/IAI.00962-08

31. Allué-Guardia A, García-Aljaro C, Muniesa M (2011) Bacteriophage-encoding cytolethal distending toxin type V gene induced from nonclinical Escherichia coli isolates. Infect Immun 79:3262-3272 . doi: 10.1128/IAI.05071-11

32. Sváb D, Bálint B, Vásárhelyi B, et al (2017) Comparative genomic and phylogenetic analysis of a Shiga toxin producing Shigella sonnei (STSS) strain. Front Cell Infect Microbiol 7:229 . doi: 10.3389/fcimb.2017.00229

33. Petty NK, Bulgin R, Crepin VF, et al (2010) The Citrobacter rodentium genome sequence reveals convergent evolution with human pathogenic Escherichia coli. J Bacteriol 192:525-538 . doi: 10.1128/JB.01144-09

\section{Legend to the figures}

Figure 1. Transmission electron micrograph of bacteriophage 130/2 showing Myoviridae morphology with contracted (A) and non-contracted (B) tail structure.

Figure 2. Whole-genome based phylogeny of bacteriophage C130_2 prepared with VICTOR, comparing it to representative members of Caudovirales, as well as bacteriophages IME_EC2 and vB_KpnS_IME279.

The GenBank accession numbers of phage genomes and type designations of the phages are indicated next to the branches. In the case of IME_EC2 and vB_KpnS_IME279, the phage families are indicated.

\section{Supplementary Table 1.}

Host spectrum and efficiency of plating (EOP) of bacteriophage C130_2. EOP values are given relative to the titre measured on $E$. coli $\mathrm{K}-12$ MG1655 strain.

\section{Supplementary Table 2.}

List of ORFs of phage C130_2 with assigned functions and protein homology searches. Prosite search was performed including motifs with high probability occurrences, Uniprot search was performed with narrowing down to viral proteins. 
217 Supplementary Table 3.

218 List of ORFs of phages C130_2, IME_EC2 and vB_KpnS_IME279, with corresponding ORFs above 75\%

219 similarity highlighted in blue.

220 Supplementary Figure 1.

221 BLAST-based comparison of the whole genomes of bacteriophages C130_2, IME_EC2 and vB_KpnS_IME279.

222 Orange arrows represent genes, numbers on C130_2 genes correspond to ORF numbers in Supplementary Tables

2232 and 3 . Regions showing $>50 \%$ similarity are interconnected with grey lines. 